\title{
Optiques de Bragg-Fresnel. Principes et applications
}

\author{
M. Idir***, A. Mirone***, G. Cauchon****** et P. Dhez**** \\ * Laboratoire de Spectroscopie Atomique et Ionique, Université Paris-Sud, 91405 Orsay, \\ France \\ ** Laboratoire pour l'Utilisation du Rayonnement Électromagnétique, Université \\ Paris-Sud, 91405 Orsay, France \\ *** Laboratoire pour l'Utilisation des Lasers Intenses, École Polytechnique, \\ 91128 Palaiseau, France \\ **** Service CEM, Section MR, CEA Bruyères-le-Châtel, 91680 Bruyères-le-Châtel, \\ France
}

\begin{abstract}
Résumé : Les lentilles de Bragg-Fresnel combinent les propriétés diffractives des réseaux et la réflexion du rayonnement $X$ des cristaux ou des multicouches. Elles permettent de réaliser des microfaisceaux et sont utilisables dans le domaine $X$ et $X-U V$. Nous rappellons ici les grands principes de ces optiques et quelques applications
\end{abstract}

\section{Introduction}

Sur toute l'étendue du spectre des ondes électromagnétiques, le domaine du visible présente une particularité essentielle pour manipuler le rayonnement. Il est le seul à posséder à la fois des matériaux extrêmement transparents, ayant en même temps des indices notables comme les diélectriques, et des corps, comme les métaux, si absorbants qu'une épaisseur d'une fraction de longueur d'onde absorbe quasiment tous les photons.

Dans le domaine des rayons $\mathrm{X}, \mathrm{l}$ 'absence de matériaux ayant des indices convenables est la cause principale des difficultés rencontrées pour les optiques. En effet, dans ce domaine d'énergie, le rayonnement est fortement absorbé et l'indice de réfraction est trés légérement inférieure à l'unité ce qui rend l'utilisation d'optiques "classiques" (miroirs en incidence normale, lentilles par réfraction, ..) quasiment impossible.

Les progrès importants récemment accomplis dans la production du rayonnement X-UV ont ouvert la voie à de nombreuses applications dans des domaines aussi divers que la radiographie, la biologie, la microscopie $X$ ou la lithographie $X, \ldots$. . Mais une bonne utilisation de ces sources nécessite le développement de nouvelles optiques faisant appel à des technologies complexes (miroirs multicouches, optiques adaptatives...) très différentes de celles employées pour les optiques traditionnelles en rayonnement visible [1].

L'emploi de miroirs en incidence rasante a longtemps été le seul moyen (avec les cristaux naturels) de focaliser le rayonnement X. Malheureusement, pour l'application à l'imagerie (microscopie), l'utilisation d'optiques en incidence rasante provoque des aberrations très importantes [2].

En 1972, E. Spiller a démontré qu'il était possible de fabriquer des miroirs interférentiels multicouches pour le domaine X-UV par dépôt sous vide alterné de deux matériaux [3]. Déposées sur des miroirs courbes, elles permettent à la fois de monochromatiser et de focaliser le rayonnement. Elles jouent un rôle de plus en plus important pour le développement de la microscopie X (incidence normale) [4] et sont envisagés pour la micro lithographie par projection.

Pour focaliser le rayonnement X-UV, une autre possibilité est d'employer des Zones de Fresnel par transmission. Elles permettent de focaliser le rayonnement incident par diffraction et jouent donc un rôle analogue à celui des lentilles du visible, qui elles fonctionnent par réfraction. 
$\mathrm{Au}$ cours des dix dernières années, les progrès de la micro lithographie [5], spécialement dans la fabrication de structure de petite taille, alliés aux techniques d'évaporation des couches minces, ont permis la réalisation de nouveaux éléments optiques : les Lentilles de BraggFresnel (LBF) [6].

Ces nouveaux éléments optiques combinent les propriétés diffractives des réseaux (gravure de type zone de Fresnel), avec la réflectivité et la sélectivité spectrale des miroirs interférentiels multicouches ou des cristaux. Ces optiques planes, analogues à des hologrammes, ce qui permet de limiter les aberrations, focalisent le rayonnement incident. La première vérification de leurs différentes propriétés avec le rayonnement synchrotron date seulement de 1986 (ZBF gravées sur un cristal de silicium) [6,7].

Dans cet article, nous rappellons les principes des optiques de Bragg-Fresnel et détaillerons quelques applications.

\section{Des miroirs pour rayons $\mathbf{X}$ au multicouches}

Par rapport aux autres domaines spectraux, celui des rayons $\mathrm{X}$ présente deux caractéristiques importantes :

a) une réfringence faible

b) une absorption notable du rayonnement sur une distance comparable à la longueur d'onde Ces deux caractéristiques conduisent à des contraintes particulières pour les optiques utilisables dans ce domaine d'énergie.

Deux solutions sont envisagées pour les optiques $\mathrm{X}[8]$ :

a) la réflexion totale externe, dans laquelle une faible épaisseur de la surface est concernée.

b) la réflexion sélective, dans laquelle une suite d'interfaces ou de plans atomiques contribue à la réflexion du rayonnement.

Etant donné la faible valeur de l'indice optique dans cette gamme de longueur d'onde, la réflexion totale externe est seulement possible sous incidence rasante.

Dans le cas des milieux périodiques, les rayons $X$ incidents sous un angle $\theta$ sont réfléchis suivant la loi de Bragg : $\mathbf{2} \mathbf{d} \sin (\theta)=\mathbf{m} \lambda$ où $d$ est la distance interéticulaire, $m$ l'ordre de diffraction, $\lambda$ la longueur d'onde du rayonnement incident. Si les périodes sont convenables, on peut ainsi réfléchir ce rayonnement par diffraction pour des angles d'incidence élevés, Cependant, pour les cristaux naturels, les atomes sont répartis sur des plans dont la périodicité n'excède pas une dizaine $d^{\prime} \AA$. On voit donc que seuls les rayonnements $X$ de courtes longueurs d'onde peuvent être réfléchis. Pour les rayons $\mathrm{X}$ mous, on peut utiliser des cristaux organiques (TIAp, KAp..) ou des couches de Langmuir Blodgett. Outre leur faible réflectivité, le grand défaut de cette catégorie d'optiques est leur faible stabilité aux flux intenses rencontrés auprès des sources synchrotron ou des plasmas. Il existe donc un réel intérêt à disposer d'optiques capables de couvrir cette gamme d'énergie.

Dans leur principe, les miroirs interférentiels adaptés aux domaines $\mathrm{X}$ et X-UV sont analogues aux multicouches diélectriques employées dans le visible, ainsi qu'aux cristaux naturels qui eux sont adaptés au domaine des $\mathrm{X}$. Ces miroirs interférentiels, préparés par évaporation. permettent de s'affranchir de la plupart des limitations rencontrées avec les optiques fonctionnant en réflexion totale, seules utilisables auparavant. Un miroir multicouche est composé d'un dépôt alterné et régulier de plusieurs couches d'épaisseur comparable à la longueur d'onde à réfléchir. Les matériaux déposés sont sélectionnés pour leur différence d'indices optiques dans le domaine de longueur d'onde considéré. Par analogie avec les cristaux dans lesquels la réflexion des rayons $\mathrm{X}$ se fait par diffraction sur les plans atomiques, 
on peut comprendre comment une série de couches d'un élément lourd (W, Si..) séparée par une couche d'un élément léger $(\mathrm{C}, \mathrm{Be}$..) est équivalent à un pseudo-cristal.

Les avancées technologiques dans la déposition des couches minces ont permis de réaliser des miroirs interférentiels multicouches de très haute qualité ayant [8] :

-- un bon pouvoir réflecteur, si l'on est capable de diminuer les rugosités

-- une faible inter-diffusion entre les différents éléments

-- une bonne stabilité aux flux très intenses

La figure 1 représente une coupe schématique d'une multicouche.

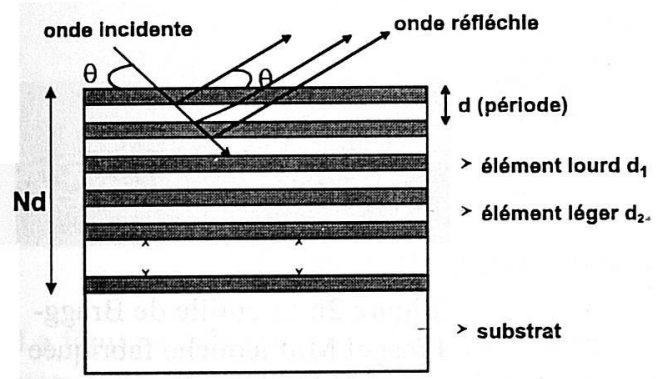

Figure 1 : Vue en coupe d'une multicouche
La longueur d'onde réfléchie est dépend directement de la périodicité du milieu réflecteur (cf. équation 5). Si l'on est donc capable de fabriquer une multicouche avec une période adaptée, on peut réfléchir n'importe quelle longueur d'onde à n'importe quel angle. En particulier, on peut également utiliser l'en incidence normale dans un important domaine de longueur d'onde ce qui est impossible avec les miroirs, qui sont limités à l'incidence rasante.

\section{Des lentilles de Fresnel au Optiques de Bragg-Fresnel}

La taille et la répartition des zones constituant les lentilles de Fresnel peuvent être expliquées à partir de l'interférence de deux points sources cohérents émettant de la lumière. La figure d'interférence produit par deux points sources S1 et S2 (cf. figure 2) est une série d'ellipsoides de révolution; l'axe de révolution étant la droite reliant les deux points sources. La figure d'interférence enregistrée sur un écran placé entre ces deux points sources donnent une lentille de Fresnel. Connu depuis trés longtemps en optique visible [9], une lentille de Fresnel est constituée d'une série de couronnes transparentes et de couronnes opaques au rayonnement incident. Les rayons des cercles varient comme les racines carrées des nombres entiers successifs $r_{n}=n^{1 / 2} r_{1}$ (figure 2) [9bis]. Avec ces lentilles, la taille du point focal est au mieux égale à la largeur de la zone externe $\left(\mathrm{dr}_{\mathrm{n}}\right)$, on cherche donc à obtenir des cercles de plus en plus fins pour augmenter la résolution. Pour obtenir une meilleure efficacité, ces lentilles sont auto portées. Elles sont donc fragiles et délicates à employer avec les flux intenses émis par un anneau synchrotron ou un plasma crée par laser. Durant les 15 dernières années, les Zones de Fresnel en transmission ont été utilisées dans la région des $\mathrm{X}$ mous tant en micro imagerie que pour la réalisation de microsondes pour des microscopes à balayage fonctionnant dans la fenêtre de l'eau (longueur d'onde de $\sim 24 \AA-32 \AA$ entre les seuils $\mathrm{K}$ de l'oxygène et du carbone) où le contraste entre l'eau et les éléments vivants est important [10].
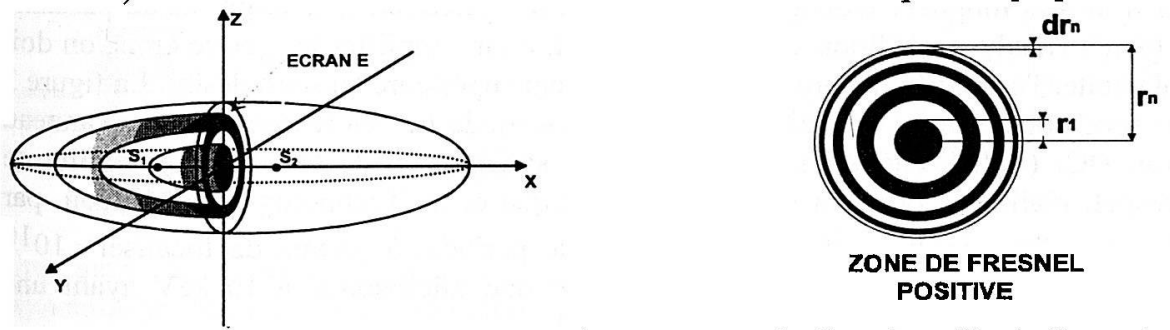

Figure 2 :Principe de fabrication et géométrie d'une Lentille de Fresnel. 
C'est à partir du principe indiquer pour les lentilles de Fresnel que V. V. Aristov et ses collaborateurs de l'Institut de micro-électronique de Moscou ont élaboré le principe des optiques de Bragg-Fresnel [6,7]. L'idée est la suivante, sur une optique réfléchissant les rayons $\mathrm{X}$, typiquement un cristal ou une multicouche, il faut réussir à créer une surface équivalente aux zones de Fresnel en transmission. Au lieu de placer un écran entre les deux points S1 et S2, on le place tangentiellement aux ellipses (figure 2a). La forme des optiques de Bragg-Fresnel à générer est plus complexe que la géométrie circulaire des lentilles de Fresnel par transmission (cf figure $2 \mathrm{~b}$ ).
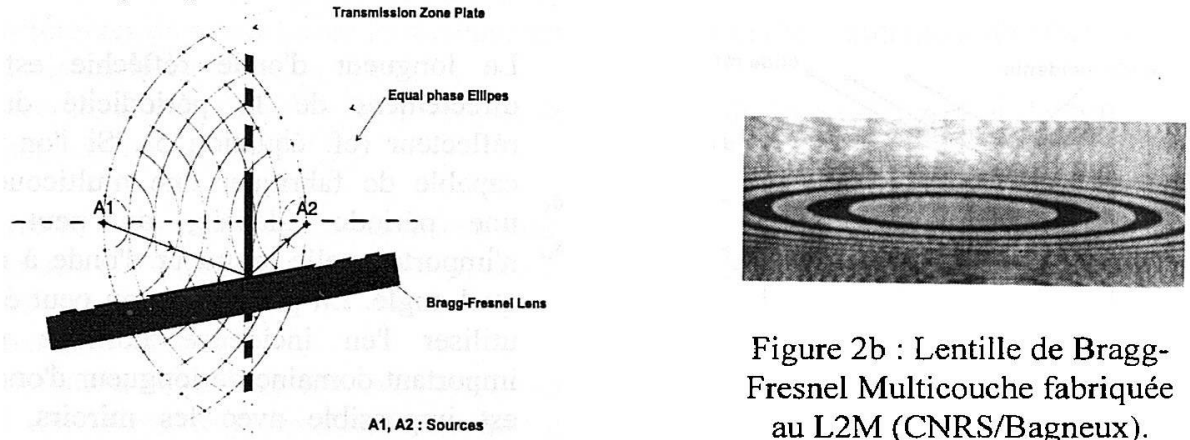

Figure $2 b$ : Lentille de BraggFresnel Multicouche fabriquée au L2M (CNRS/Bagneux).

Interference pattern from two spherical waves

Figure 2a : Principe de fabrication des lentilles de Fresnel

et de Bragg-Fresnel

Comme on peut le remarquer, on obtient une famille d'ellipses décentrées et surtout très allongées dans la direction du rayonnement incident car l'angle d'attaque $\theta$ reste très faible pour la réflexion des rayons $\mathrm{X}$ sur des multicouches (quelques degrés). Le rapport entre les deux axes d'une ellipse est : $\sin (\theta)$.

Pour ces nouvelles optiques, on a employé les microtechnologies jusqu'alors développées dans l'industrie des semi-conducteurs pour la fabrication des circuits intégrés :masqueur électronique, bâti de gravure (plamas ou chimique).

\section{Optiques de Bragg-Fresnel: Applications}

La principale application des LBF est la possibilité de focaliser un faisceau de rayons X ce qui permet de réaliser des microsondes $X$. Pour le domaine vers $0,1 \mathrm{~nm}$, la géométrie la plus simple est celle d'une lentille de Fresnel à zones circulaires fonctionnant par réflexion de Bragg à partir d'un"cristal. Dans ce cas il faut travailler sous incidence normale en retour. Pour les optiques de Bragg-Fresnel usinées sur du silicium comme réflecteur, cette solution fut la premiere testée à l'aide d'une source synchrotron [11]

Comme on l'a vu dans la première partie, l'utilisation de miroirs multicouches offre la liberté de choisir à la fois longueur d'onde et l'incidence. En particulier, leur large bande passante convient bien à l'analyse par fluorescence $\mathrm{X}$. En effet, pour simplifier le spectre émis, on doit seulement exciter l'échantillon à une énergie légèrement supérieure au seuil désiré. La figure 3 montre le spot obtenu pour la réalisation d'une microsonde de fluorescence $\mathrm{X}$ sur l'anneau synchrotron DCI (LURE/ORSAY). Avec la source synchrotron de l'ESRF, une lentille de Bragg Fresnel, réalisée à l'Institut de Microélectronique et de Technologie de Moscou, par attaque ionique d'une multicouche W/Si de $5 \mathrm{~nm}$ de période, à permis de focaliser $10^{10}$ photons / sec dans quelques microns et de réaliser une microsonde à $10 \mathrm{keV}$ ayant une résolution spatiale jamais obtenue à cette énergie [12]. 

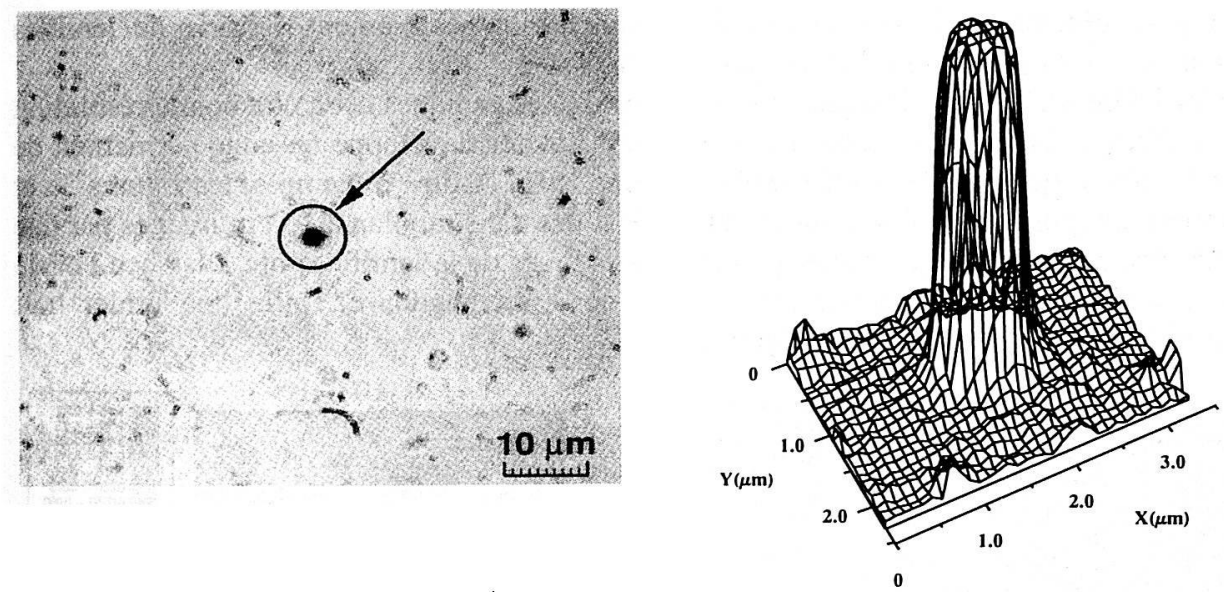

Figure 3 : Point focal obtenu sur l'anneau synchrotron DCI

La figure 4 schématise l'un des problèmes rencontrés avec des lentilles de Bragg-Fresnel, et une solution employée pour le résoudre. Dans toute lentille "centrée" travaillant par diffraction en transmission ou en réflexion, les différentes longueurs d'onde et les ordres successifs sont focalisés le long de l'axe. A la distance focale correspondant à l'ordre utilisé, ceci apparaît comme un halo diminuant la résolution spatiale dans les applications. La figure du haut montre que l'ordre zéro apparaît comme un cercle; c'est la projection de la surface de la lentille après réflexion spéculaire. Pour augmenter le contraste autour du point de focalisation de l'ordre 1, on doit minimiser la contribution de l'ordre zéro. Une première approche consiste à réaliser une lentille de phase en ajustant la profondeur de la gravure lors de l'attaque ionique.

On peut aussi penser à une autre propriété des réseaux. Le réseau étant l'équivalent d'un hologramme, on peut penser à en utiliser une surface égale, mais prise en dehors de l'axe. Pour une telle lentille dite "hors axe", l'ordre zéro est toujours réfléchi en réflexion symétrique. Par contre, dans le plan focal de l'ordre 1, il se trouve alors spatialement séparé du rayonnement focalisé en ordre 1 puisque ce dernier reste focalisé sur l'axe de l'hologramme initial.

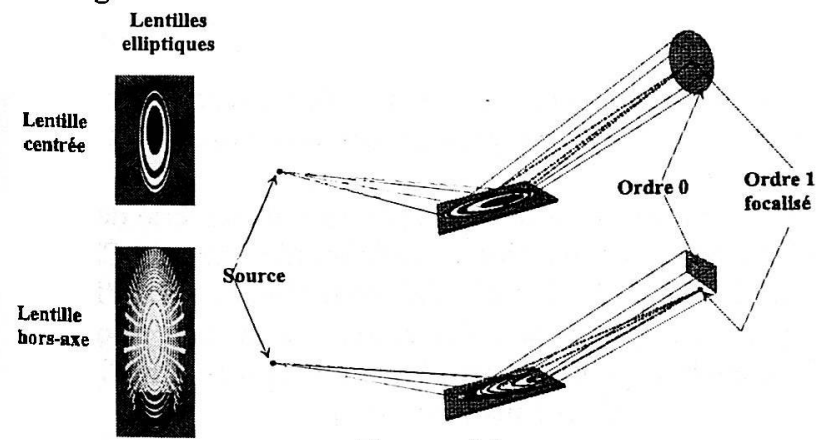

Figure $n^{\circ} 4$

On remarquera que le contour et l'emplacement de la lentille horsaxe sont choisis dans l'hologramme pour éviter d'avoir à réaliser des traits trop fins. La validité de ce raisonnement à été confirmé par le calcul [13]. Pour vérifier la faisabilité de ces nouvelles lentilles hors-axe, des images tests ont été obtenues autour de $1 \mathrm{keV}$ [14].

Une autre application possible des LBF est l'imagerie de sources de rayonnement X (synchrotron, plasmas,..). Par exemple, elles sont employées à l'ESRF (Grenoble) et à Bessy 
(Berlin) pour obtenir en $\mathrm{X}$ les images des onduleurs respectivement à l'aide de lentilles gravées sur silicium ( $9 \mathrm{keV}$ ) [15] et sur multicouche (190 eV) [16].

La possibilité de réaliser des images de sources émissives étendues avec une bonne résolution spatiale à d'abord été étudiée théoriquement ${ }^{1}$. Nous avons développé un code permettant de calculer les aberrations sur le point focalisé par une LBF. A titre d'exemple, les figures $5 a$ et $5 \mathrm{~b}$ montrent la réponse de points sources émissifs situés dans un plan à $15 \mathrm{~cm}$ imagés par une lentille de Bragg-Fresnel multicouche (les caractéristiques de la lentille sont : Distance Focale $15 \mathrm{~cm}$, Ouverture : $200 \mu \mathrm{m}$ par $200 \mu \mathrm{m}$ (Lentille rectangulaire), Taille du dernier trait (résolution limite) $0.5 \mu \mathrm{m}$ (200 traits gravés).
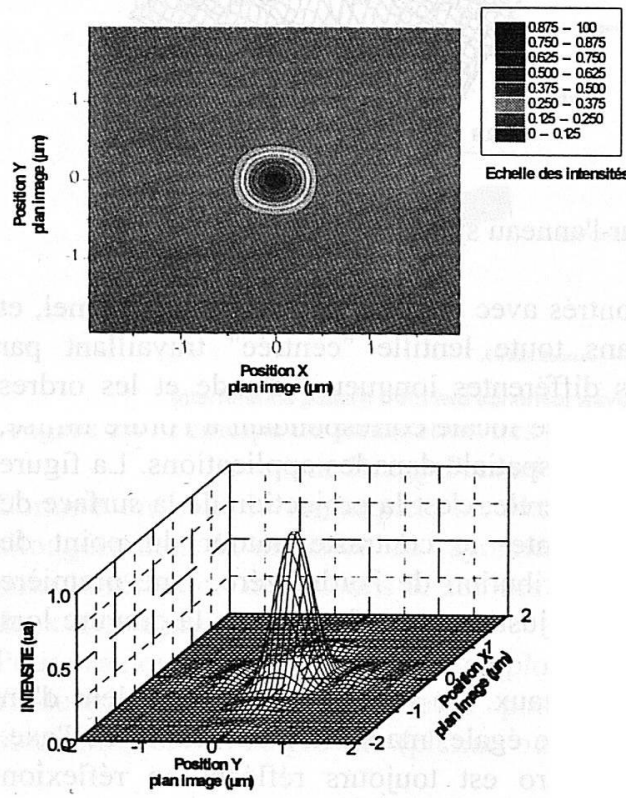

Figure 5à : Image d'un point source situé sur l'axe optique
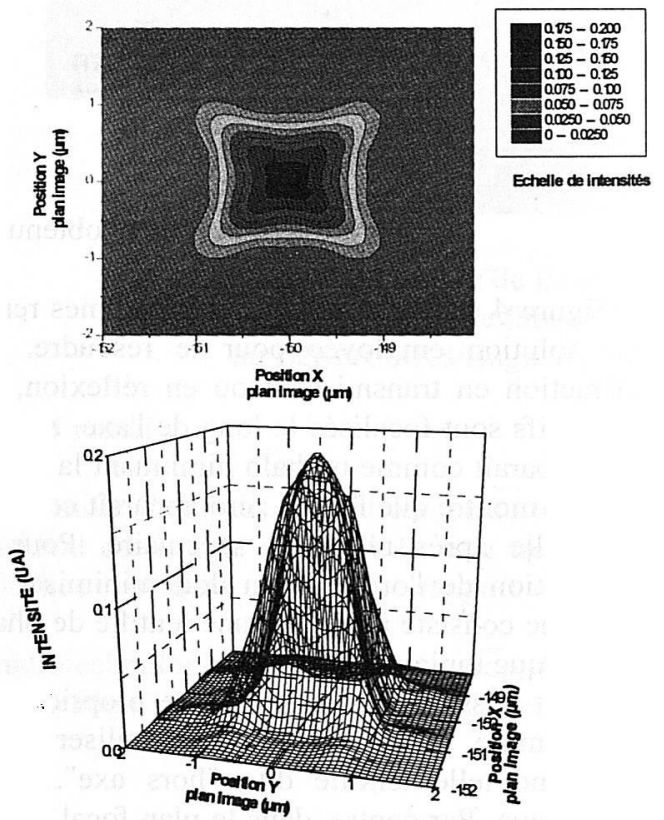

Figure 5b: Image d'un point source déplacé de 1 milliradian suivant la direction $\mathrm{X}$ par rapport à l'axe optique.

On voit les effets des aberrations géométriques sur la résolution spatiale lorsque l'on déplace ce point source dans le plan objet. Les distances p et q représentent respectivement la distance source-lentille et lentille-plan image.

Ce type d'étude a permis de dimensionner une lentille pour une expérience d'imagerie de grille avec un plasma créé par laser. Cette expérience a été réalisée en collaboration avec le CEA de Bruyères le Châtel (Service CEM) et le CEA de Limeil Valenton (Service DLPP) sur l'installation OCTAL. La figure 6 montre l'image de la grille obtenue avec une résolution spatiale de l'ordre de 4-5 microns. Cette étude en cours de dévelopement et vise à réaliser un dispositif d'imagerie d'instabilité auprés des plasmas créé par laser. [17].

\footnotetext{
'Cette étude a été menée en collaboration avec le CEA de Bruyères le Châtel (Service CEM/MR) par le biais du contrat $n^{\circ} \mathrm{U} 44002598 \mathrm{IL}$
} 


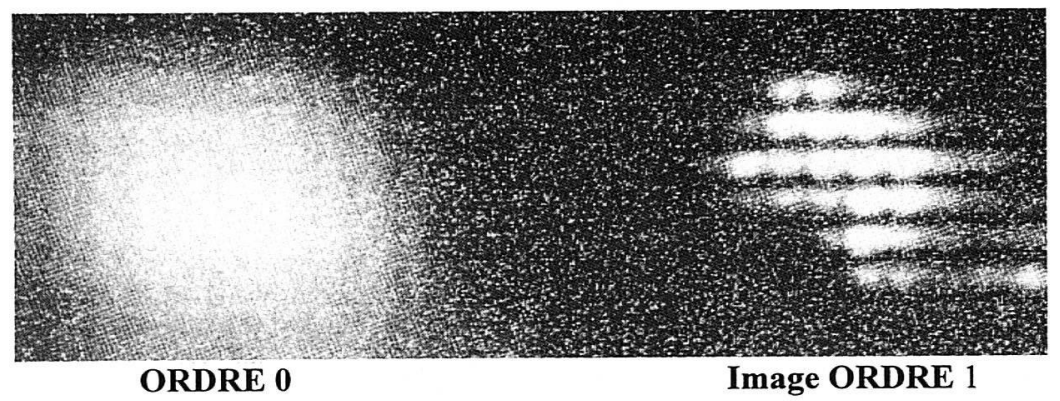

Figure 6 : Image de grille obtenue avec une lentille de Bragg-Fresnel Multicouche sur le laser Octal $(\mathrm{E}=1430 \mathrm{eV})$

\section{Conclusion}

Dans les rayons $\mathrm{X}$, les lentilles basées sur la réfraction n'existant pas, et les miroirs classiques étant limités à l'incidence très rasante, les optiques par diffraction (Lentilles de Bragg-Fresnel et Lentillesde Fresnel) représentent une nouvelle voie pour les optiques X.

Dévelopées depuis prés de 10 ans, les LBF montrent des potentialités importantes tant dans l'imagerie de plasmas que dans leur utilisation auprés des sources synchrotron dites de 3ème génération pour la réalisation de microfaisceau qui peut être ensuite utilisée pour des expériences aussi diverses que : la microfluorescence, la microdiffraction, la microscopie,..

\section{Remerciements}

Les auteurs remercient P. Chevallier (LURE), F. Legrand (ESRF), A. Erko (Bessy), le CEA de Bruyères le Châtel (Service CEM Section MR) et de Limeil Brevannes (Service DLPP Section ED) pour leur aide.

\section{Références}

[1] J.H. Underwood, D.T. Attwood, "The renaissance of X-ray optics" Phys. Today April $1984,44-52$

[2] A. Franck, "X-Ray Optics", Sci. Prog. Oxf. (1977) 64,371-422

[3] E. Spiller, Appl. Phys. Lett. 20,1972, Applied Optics 16,89,1976.

[4] P. Dhez, "X and X-UV Microscopes Based on Mirror Optics"

X-ray Microscopy III, London Sept 3-7 1990 ed. A. Michette (Springer Verlag)

[5] C. Khan Malek"A review of Microfabrication Technologies. Application to X-ray Optics" Journal of X-ray Science and Technology vol 3, ${ }^{\circ} 1$, Oct. 1993, 45-68

[6] V.V. Aristov, A.A. Snigirev, Yu A. Basov "X-ray Bragg Optics" Proceedings of the conference on short wavelength Coherent radiation Generation and Applications (Monterrey USA 1986)

[7] V. V. Aristov, S.V. Gaponov et al ."Focussing properties of profiled $x$-ray multilayer mirrors" JETP Lett. 44, 265-267, 1986 
[8] P. Dhez, "Progress in multilayer devices as X-ray optical elements" J. Microscopy 138,(3),267,(1985)

[9] J.L. Soret, "Concerning diffraction by circular gratings" Arch. Sci. Phys. Nat. 52,320,(1875)

[9bis] A. G. Michette, "Optical system for soft X-rays" Plenum Press, New York 1986

[10] X-ray Microscopy I ed. G. Schamhl, D. Rudolph (Springer Verlag Berlin 1984) X-ray Microscopy II, ed. D. Sayre, M. Howells, J. Kirz, H. Rarback (Springer Verlag 1988) X-ray Microscopy III, London Sept 3-7 1990 ed. A. Michette (Springer Verlag)

[11] Yu Basov, A . Snigirev, P. Dhez et al.

"Two dimensionnal focussing of hard $x$-ray by a phase circumar bragg-Fresnel Lens" NIM A308,p 363 (1991.

[12] P. Chevallier, P. Dhez, F. Legrand, M. Idir, G. Soullie, A. Mirone, A. Erko, A. Snigirev, I. Snigireva, A. Suvorov, A.Freund, A. Grubel

"Scanning x-ray microprobe with Bragg-Fresnel lens at ESRF beam line" NIM A354, 584-587, 1995

[13] A. Mirone, M. Idir, G. Soullie, P. Dhez, A. Erko "Dynamical theory for Bragg-Fresnel multilayer lenses for $X$-UV and X-ray range" Optics Communications, $111,191,(1994)$

[14] M. Idir

"Etude expérimentale et théorique d'optiques focalisantes de types Bragg-Fresnel gravées sur des multicouches adaptées aux domaines $X$ et $X-U V^{\prime \prime}$

Thèse Université Pierre et Marie Curie (Paris VI) (1994)

A. Mirone, M. Idir, G. Soullié, P. Guerin , F.R. Ladan, P. Dhez, H. Launois

"New design for Bragg-Fresnel Multilayer lens" X-ray Microscopy IV (Moscou Sept. 93)

[15] E. Tarazona, P. Elleaume, J. Chavanne, YA. M. Hartman, A. A. Snigirev,I. I. Snigireva " $2 D$ imaging of an undulator source by phase circular Bragg-Fresnel lens" Rev. Sci. Instrum. 65 (6) 1994

[16] K. Holldack, A. Erko, W.B. Peatman, T. Noll

"Multilayer Bragg-Fresnel Monitor" Bessy Newsletter n²4 p.3, 1994.

[17] G. Cauchon "Imagerie de plasma à l'aide de Lentilles de Bragg-Fresnel" en cours 